\title{
Motion of vortices in ferromagnetic spin-1 BEC
}

\author{
E. B. Sonin \\ Racah Institute of Physics, Hebrew University of Jerusalem, \\ Givat Ram, Jerusalem 91904, Israel
}

(Dated: March 20, 2018)

\begin{abstract}
The paper investigates dynamics of nonsingular vortices in a ferromagnetic spin-1 BEC, where spin and mass superfluidity coexist in the presence of uniaxial anisotropy (linear and quadratic Zeeman effect). The analysis is based on hydrodynamics following from the Gross-Pitaevskii theory. Cores of nonsingular vortices are skyrmions with charge, which is tuned by uniaxial anisotropy and can have any fractal value between 0 and 1 . There are circulations of mass and spin currents around these vortices. The results are compared with the equation of vortex motion derived earlier in the Landau-Lifshitz-Gilbert theory for magnetic vortices in easy-plane ferromagnetic insulators. In the both cases the transverse gyrotropic force (analog of the Magnus force in superfluid and classical hydrodynamics) is proportional to the charge of skyrmions in vortex cores.
\end{abstract}




\section{INTRODUCTION}

Similarly to superfluid ${ }^{3} \mathrm{He}$, the ferromagnetic spin-1 BEC combines properties of a common superfluid and of a magnetically ordered system [1]. Correspondingly, one may expect coexistence and interplay of spin superfluidity [2, 3] and more common mass superfluidity. Like common ferromagnets, the spin-1 BEC is described at macroscopical scales by the Landau-Lifshitz-Gilbert (LLG) theory [4] but extended by inclusion of an additional degree of freedom of fluid motion as a whole [5, 6].

Emergence of superfluidity is conditioned by special topology of the order parameter space. In the case of scalar superfluids the order parameter space is a circumference on a complex plane of a complex wave function. Topology of the circumference allows mass superfluidity, since current states map on paths winding around the circumference. These mappings cannot be reduced to a point by continuous transformation without leaving the circumference. As for spin superfluidity in magnetically ordered media, this requires the easy-plane anisotropy in the spin space [2, 3]. This anisotropy can emerge not only from crystal anisotropy, but also from long-range magnetostatic (dipolar) interaction as shown for the magnon condensate in yttrium-iron-garnet magnetic films [7].

Manifestation of superfluidity is macroscopic persistent currents proportional to gradients of phase (phase of the wave function for mass superfluidity and the angle of spin rotation around some axis in the case of spin superfluidity). Persistent current states are metastable states, but they lose stability when phase gradients reach some critical values. After this frequent phase slips destroy persistent currents and relaxation to current-less ground states occurs. Vortices also emerge in the equilibrium rotating superfluids. Thus vortices are crucial for the phenomenon of superfluidity. Its very existence as linear topological defects requires the same topology as necessary for existence of superfluidity.

Investigation of vortices in scalar superfluids started from the seminal works of Onsager [8] and Feynman [9]. In magnetically ordered systems magnetic vortices also were known long ago. Magnetic vortex is an example of topological defects in magnetically ordered solids, which were in the focus of scientific activity of Arnold Markovich Kosevich and his colleagues [10 12]. At motion of a magnetic vortex a reactive gyrotropic force proportional and normal to its velocity emerges. This gyrotropic force was first revealed by Thiele [13] for magnetic bubbles in ferromagnetic films. Later the gyroscopic force was derived for magnetic 
vortices in easy-plane magnets [14, 15]. In contrast to friction force also proportional to the vortex velocity, the gyrotropic force does not depend on the spin texture inside the vortex core, but does depend on circulation of the spin phase (the angle of spin rotation in the easy-plane).

The goal of the present paper is derivation of the equation of motion of a nonsingular vortex in the ferromagnetic spin-1 BEC. Nonsingular vortices are possible in multi-component superfluids when vorticity is not concentrated at a singular line (axis of the vortex) but is continuously distributed over a core of finite radius. First they were revealed in the $A$ phase of superfluid ${ }^{3} \mathrm{He}$ [16]. The energy of nonsingular vortices is smaller than of singular ones, and phase slips with nonsingular vortices are more probable. In the ferromagnetic spin-1 BEC mass and spin superfluidity coexist, and a nonsingular vortex is a hydrodynamic and a magnetic vortex at the same time, i.e., it has circulations of mass and spin currents around it. This has an impact on phase slips destroying mass and spin supercurrent [6]. Normally the core radius of nonsingular vortices exceeds microscopical scales, and the hydrodynamical approach is sufficient for derivation of the equation of vortex motion.

There are two methods to derive the equation of vortex motion. The first one is using the solvability condition. The hydrodynamic equations (the LLG equations in the case of magnetic vortices) are linearized with respect to small perturbations of the static solution for a resting vortex. Perturbations are produced by vortex motion and by currents past the vortex. This yields nonuniform linear equations. It is not necessary to solve the equations explicitly. The equation of vortex motion is derived from a condition for their solvability, which is called also the condition of the absence of secular terms. This approach was used in the past for the analysis of dynamics of nonsingular vortices in ${ }^{3} \mathrm{He}-A$ [17]. The second method uses the conservation law for momentum in a Galilean invariant medium, or for quasimomentum if Galilean invariance is broken following from Noether's theorem for translationally invariant media. In the absence of external forces or friction force, which violate the conservation law for (quasi)momentum, the second method yields exactly the same equation of vortex motion as the first method [15]. Here we use the second method. The equation of motion is the balance equation of forces on the vortex. There are a gyrotropic force proportional to the vortex velocity (analog of the Magnus force on a hydrodynamic vortex) and a force proportional to mass and spin supercurrents past the vortex (Lorentz force in superfluid hydrodynamics). These forces depend on topological charges of vortices 
but not on details of the core structure, in contrast to the friction force, which does depend on details of the vortex core but is not investigated in the present work.

We start our analysis from formulation of hydrodynamics of ferromagnetic spin-1 BEC following from the Gross-Pitaevskii theory (Sec. II). Section III reviews dynamics of magnetic vortices in ferromagnet insulators, where mass (charge) currents are absent. This is necessary for comparison with dynamics of vortices in the ferromagnetic spin-1 BEC, where both mass and spin currents are possible. Sections II and III review previously known results. Dynamics of vortices in the ferromagnetic spin-1 BEC is addressed in Sec. IV] Discussion and conclusions are presented in Sec. V.

\section{GROSS-PITAEVSKII THEORY FOR FERROMAGNETIC SPIN-1 BEC}

In the spin-1 ferromagnetic BEC the condensate wave function (the order parameter) can be presented as a $3 \mathrm{D}$ complex vector in the spin space:

$$
\boldsymbol{\psi}=\frac{\psi_{0}}{\sqrt{2}}(\boldsymbol{m}+i \boldsymbol{n})
$$

where scalar $\psi_{0}$ and two unit mutually orthogonal vectors $\boldsymbol{m}$ and $\boldsymbol{n}$ are real. The two unit vectors $\boldsymbol{m}$ and $\boldsymbol{n}$ together with the third vector

$$
s=\boldsymbol{m} \times \boldsymbol{n}
$$

form a triad of three real orthogonal unit vectors. The unit vector $s$ points out direction

of full spin polarization. It is an analog of the orbital vector $\boldsymbol{l}$ in the $A$ phase of superfluid 3He, which shows direction of the orbital moment of Cooper pairs. Neutral and charged superfluids with such order parameter are called chiral or $p_{x}+i p_{y}$ superfluids.

The gauge transformation of the ferromagnetic spin-1 order parameter,

$$
\boldsymbol{m}+i \boldsymbol{n} \rightarrow(\boldsymbol{m}+i \boldsymbol{n}) e^{i \theta}=(\boldsymbol{m} \cos \theta-\boldsymbol{n} \sin \theta)+i(\boldsymbol{m} \sin \theta+\boldsymbol{n} \cos \theta)
$$

is equivalent to rotation around the axis $s$ by the angle $\phi_{s}=-\theta$ and therefore is not an independent symmetry transformation. So the full point symmetry group of the order parameter is the group $S O(3)$ of three-dimensional rotations. The group is not abelian, and the angle of rotation around any axis including the axis $s$ depends on the path along which the transformation is performed. In particular, if we deal with the phase $\theta=-\phi_{s}$, a result 
of two small consecutive variations $\delta_{1}$ and $\delta_{2}$ of $\theta$ depends on the order of their realizations:

$$
\delta_{1} \delta_{2} \theta-\delta_{1} \delta_{2} \theta=\boldsymbol{s} \cdot\left[\delta_{1} \boldsymbol{s} \times \delta_{2} \boldsymbol{s}\right]
$$

This means that the phase $\theta$ is not well defined globally, although its infinitesimal variations still make sense, and the quantum-mechanical definition of the superfluid velocity,

$$
\boldsymbol{v}_{s}=\frac{\hbar}{m} \nabla \theta
$$

is valid. Here $m$ is the mass of a boson. Because of Eq. (4) variation of the superfluid velocity is determined not only by variation of the phase $\theta$ itself but also by variation of the spin vector $\boldsymbol{s}$. As a result, the superfluid velocity is not curl-free. Replacing $\delta_{1}$ and $\delta_{2}$ in Eq. (4) with two gradients $\nabla_{1}$ and $\nabla_{2}$ along two different directions ( $x$ and $y$, or $y$ and $z$, or $z$ and $x$ ), Eq. (4) yields the Mermin-Ho relation [18] between vorticity and spatial variation of $s$ :

$$
\boldsymbol{\nabla} \times \boldsymbol{v}_{s}=\frac{\hbar}{2 m} \epsilon_{i k n} s_{i} \boldsymbol{\nabla} s_{k} \times \nabla s_{n}
$$

This relation has a dramatic impact on hydrodynamics of chiral superfluids.

For bosons with spin 1 the most general Lagrangian of the Gross-Pitaevskii theory is

$$
\mathcal{L}=\frac{i \hbar}{2}\left(\boldsymbol{\psi}^{*} \cdot \frac{\partial \boldsymbol{\psi}}{\partial t}-\boldsymbol{\psi} \cdot \frac{\partial \boldsymbol{\psi}^{*}}{\partial t}\right)-H
$$

where $H$ is the Hamiltonian, which can depend on the wave function $\boldsymbol{\psi}$ and its gradients.

According to Noether's theorem, gauge invariance leads to the mass continuity equation:

$$
\frac{\partial \rho}{\partial t}+\nabla \cdot \boldsymbol{j}=0
$$

where

$$
\rho=\frac{1}{i \hbar}\left(\frac{\partial \mathcal{L}}{\partial \dot{\boldsymbol{\psi}}} \cdot \boldsymbol{\psi}-\frac{\partial \mathcal{L}}{\partial \dot{\boldsymbol{\psi}}^{*}} \cdot \boldsymbol{\psi}^{*}\right)=m \boldsymbol{\psi}^{*} \cdot \boldsymbol{\psi}
$$

is the mass density and

$$
\boldsymbol{j}=\frac{1}{i \hbar}\left(\frac{\partial \mathcal{L}}{\partial \boldsymbol{\nabla} \psi_{j}} \psi_{j}-\frac{\partial \mathcal{L}}{\partial \boldsymbol{\nabla} \psi_{j}^{*}} \psi_{j}^{*}\right)
$$

is the mass current.

Noether's theorem connects translational invariance with the conservation law

$$
\frac{\partial g_{i}}{\partial t}+\nabla_{j} \Pi_{i j}=0,
$$


where

$$
\boldsymbol{g}=-\frac{\partial \mathcal{L}}{\partial \dot{\psi}_{j}} \boldsymbol{\nabla} \psi_{j}-\frac{\partial \mathcal{L}}{\partial \dot{\psi}_{j}^{*}} \boldsymbol{\nabla} \psi_{j}^{*}=-\frac{i \hbar}{2}\left(\psi_{j}^{*} \nabla \psi_{j}-\psi_{j} \boldsymbol{\nabla} \psi_{j}^{*}\right)
$$

is a current, which can be different from the mass current in general, and

$$
\Pi_{i j}=-\nabla_{i} \psi_{k} \frac{\partial \mathcal{L}}{\partial \nabla_{j} \psi_{k}}-\nabla_{i} \psi_{k}^{*} \frac{\partial \mathcal{L}}{\partial \nabla_{j} \psi_{k}^{*}}+\delta_{i j} \mathcal{L}
$$

is some flux tensor.

The third conservation law follows from Noether's theorem if the Hamiltonian is invariant with respect to any rotation in the spin space:

$$
\frac{\partial S_{i}}{\partial t}+\nabla_{j} J_{i j}=0
$$

where

$$
\boldsymbol{S}=-\frac{\partial \mathcal{L}}{\partial \dot{\boldsymbol{\psi}}} \times \boldsymbol{\psi}-\frac{\partial \mathcal{L}}{\partial \dot{\boldsymbol{\psi}}^{*}} \times \boldsymbol{\psi}^{*}=i \hbar\left[\boldsymbol{\psi} \times \boldsymbol{\psi}^{*}\right]=\frac{\hbar \rho}{m} \boldsymbol{s}
$$

is the spin density and

$$
J_{i j}=-\epsilon_{i j k}\left(\frac{\partial \mathcal{L}}{\partial \nabla_{j} \boldsymbol{\psi}} \cdot \nabla_{k} \boldsymbol{\psi}+\frac{\partial \mathcal{L}}{\partial \nabla_{j} \boldsymbol{\psi}^{*}} \cdot \nabla_{k} \boldsymbol{\psi}^{*}\right)
$$

is the spin current tensor.

If the BEC is Galilean invariant as it should be in the absence of optical lattices, the Hamiltonian and the nonlinear Schrödinger equation are

$$
\begin{gathered}
H=\frac{\hbar^{2}}{2 m} \nabla_{i} \psi_{j}^{*} \nabla_{i} \psi_{j}+\frac{V|\boldsymbol{\psi}|^{4}}{2} \\
i \hbar \frac{\partial \boldsymbol{\psi}}{\partial t}=\frac{\delta H}{\delta \boldsymbol{\psi}^{*}}=-\frac{\hbar^{2} \nabla_{j}^{2} \boldsymbol{\psi}}{2 m}+V|\boldsymbol{\psi}|^{2} \psi .
\end{gathered}
$$

Only for a Galilean invariant superfluid the current $\boldsymbol{g}$ coincides with the mass current $\boldsymbol{j}$, which at the same time is the momentum density of the superfluid. Then the conservation law (11) is the conservation of the momentum, and the flux tensor

$$
\Pi_{i j}=\frac{\hbar^{2}}{2 M}\left(\nabla_{i} \psi_{k} \nabla_{j} \psi_{k}^{*}+\nabla_{i} \psi_{k}^{*} \nabla_{j} \psi\right)+\delta_{i j} P
$$

is the momentum flux tensor with the pressure given by

$$
P=\mathcal{L}=\frac{V|\boldsymbol{\psi}|^{4}}{2}-\frac{\hbar^{2}}{4 m} \nabla^{2}|\boldsymbol{\psi}|^{2}
$$

In the absence of Galilean invariance we shall call the current $\boldsymbol{g}$ the quasimomentum density and the tensor $\Pi_{i j}$ the quasimomentum flux tensor. If the superfluid is in a periodic potential 
(BEC in an optical lattice, e.g.) the current $\boldsymbol{g}$ is a density of the quasimomentum indeed as it is defined in the Bloch band theory [19]. The Gross-Pitaevskii theory for $p_{x}+i p_{y}$ superfluids, which is presented here, has already been used in the past for the $A$ phase of superfluid ${ }^{3} \mathrm{He}$ [19, 20].

Transition from the Gross-Pitaevskii theory to the hydrodynamical description is realized by the generalized Madelung transformation. After the transformation the superfluid is described by the mass density $\rho=m \psi_{0}^{2}$, the orbital vector $\boldsymbol{s}$, and the quantum-mechanical phase $\theta$. In the hydrodynamical approach usually they neglect dependence of the energy on density gradients (gradients of $\psi_{0}$ ) responsible for quantum pressure [19]. The Lagrangian and the Hamiltonian after the Madelung transformation become

$$
\begin{gathered}
\mathcal{L}=-\frac{\hbar}{m} \rho \frac{\partial \theta}{\partial t}-H \\
H=\frac{\rho}{2} v_{s}^{2}+\frac{\hbar^{2} \rho}{4 m^{2}} \nabla_{i} \boldsymbol{s} \cdot \nabla_{i} \boldsymbol{s}+\frac{V \rho^{2}}{2 m^{2}} .
\end{gathered}
$$

In hydrodynamics two canonical equations of motion are the continuity equation (8) and the Josephson equation for the phase $\theta$,

$$
\frac{\hbar}{m} \frac{\partial \theta}{\partial t}+\mu_{0}+\frac{v_{s}^{2}}{2}=0
$$

They are similar to those in a non-chiral superfluid. Here

$$
\mu_{0}=\frac{\hbar^{2}}{4 m^{2}} \nabla_{i} \boldsymbol{s} \cdot \nabla_{i} \boldsymbol{s}+V \rho
$$

is the chemical potential of the superfluid at rest.

The third hydrodynamical equation is for the unit vector $s$ :

$$
\frac{\partial \boldsymbol{s}}{\partial t}+\left(\boldsymbol{v}_{s} \cdot \nabla\right) \boldsymbol{s}-\frac{\hbar}{2 m \rho}\left[\boldsymbol{s} \times \nabla_{i}\left(\rho \nabla_{i} \boldsymbol{s}\right)\right]=0 .
$$

For a fluid at rest $\left(\boldsymbol{v}_{s}=0\right)$ Eq. (25) is identical to the LLG equation for magnetization in a solid ferromagnetic insulator.

After the Madelung transformation the spin current tensor 16 becomes

$$
J_{i j}=S_{i} v_{s j}-\epsilon_{i k l} s_{k} \frac{\partial H}{\partial \nabla_{j} s_{l}}=S_{i} v_{s j}-\frac{\hbar^{2} \rho}{2 m^{2}} \epsilon_{i k l} s_{k} \nabla_{j} s_{l} .
$$


The first term in the expression for the spin current presents advection of spin by fluid motion as a whole. This effect is trivial and has nothing to do with special conditions required for existence of spin superfluidity. Only the second term,

$$
j_{i j}=-\frac{\hbar^{2} \rho}{2 m^{2}} \epsilon_{i k l} s_{k} \nabla_{j} s_{l}
$$

connected with stiffness of the spin texture will be later called the spin supercurrent.

The Euler equation for the velocity $\boldsymbol{v}_{s}$ must follow from the Josephson equation (23) by applying the gradient operator. But one should take into account non-commutativity of the operators $\partial / \partial t$ and $\boldsymbol{\nabla}$ at their actions on the phase $\theta$. Namely, according to Eq. (4)

$$
\nabla_{i} \frac{\partial \theta}{\partial t}-\frac{\partial\left(\nabla_{i} \theta\right)}{\partial t}=\nabla_{i} \frac{\partial \theta}{\partial t}-\frac{m}{\hbar} \frac{\partial v_{s i}}{\partial t}=\boldsymbol{s} \cdot\left[\nabla_{i} \boldsymbol{s} \times \frac{\partial \boldsymbol{s}}{\partial t}\right]
$$

After some algebra using the Mermin-Ho relation (6) and the equation (25) of spin dynamics one obtains the Euler equation

$$
\dot{\boldsymbol{v}}_{s}+\left(\boldsymbol{v}_{s} \cdot \boldsymbol{\nabla}\right) \boldsymbol{v}_{s}+\nabla \mu_{0}+\frac{\hbar^{2}}{2 m^{2}} \nabla s_{i} \frac{\nabla_{j}\left(\rho \nabla_{j} s_{i}\right)}{\rho}=0 .
$$

It is possible to avoid dealing with the globally undefined phase $\theta$ by introducing Euler angles as hydrodynamical variables. They determine rotation of the triad $\boldsymbol{m}, \boldsymbol{n}, \boldsymbol{s}$ with respect to the original triad $\hat{x}, \hat{y}, \hat{z}$ as shown in Fig. 1 ;

$$
\begin{array}{ccc}
m_{x}=\cos \beta \cos \alpha \cos \varphi-\sin \alpha \sin \varphi, & m_{y}=\cos \beta \cos \alpha \sin \varphi+\sin \alpha \cos \varphi, & m_{z}=-\sin \beta \cos \alpha \\
n_{x}=-\cos \beta \sin \alpha \cos \varphi-\cos \alpha \sin \varphi, & n_{y}=-\cos \beta \sin \alpha \sin \varphi+\cos \alpha \cos \varphi, & n_{z}=\sin \beta \sin \alpha \\
s_{x}=\sin \beta \cos \varphi, & s_{y}=\sin \beta \sin \varphi, & s_{z}=\cos \beta
\end{array}
$$

In terms of the Euler angles the superfluid velocity is

$$
\boldsymbol{v}_{s}=-\frac{\hbar}{m}(\boldsymbol{\nabla} \alpha+\cos \beta \boldsymbol{\nabla} \varphi)
$$

while the Mermin-Ho relation becomes

$$
\left[\boldsymbol{\nabla} \times \boldsymbol{v}_{s}\right]=\frac{\hbar}{m} \sin \beta[\nabla \beta \times \nabla \varphi]
$$

According to Eq. (27), the current of the $z$-component of spin is

$$
\boldsymbol{j}_{z}=-\frac{\hbar^{2} \rho}{2 m^{2}} \sin ^{2} \beta \boldsymbol{\nabla} \varphi
$$




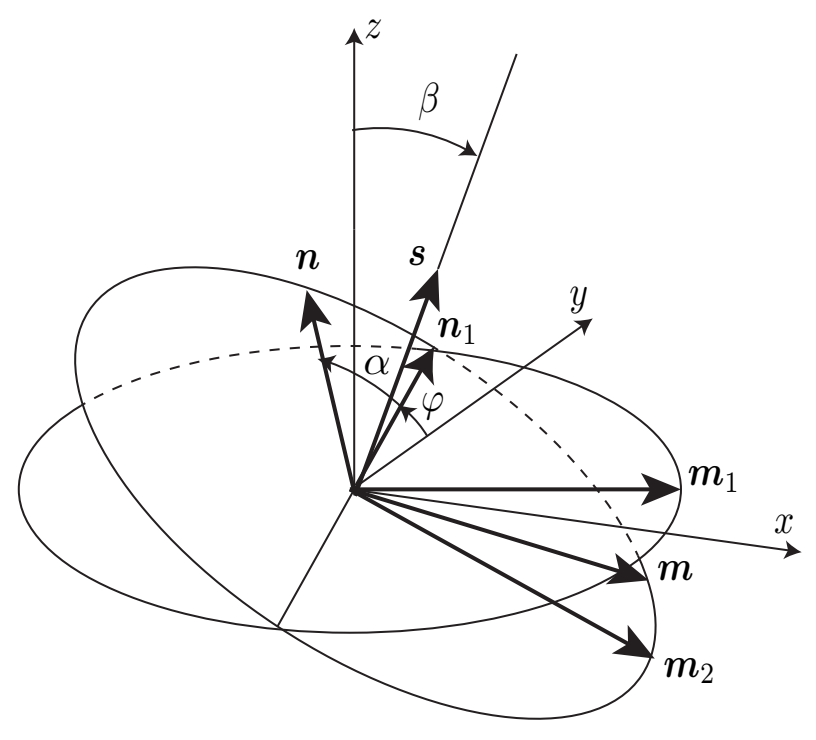

FIG. 1. Euler angles for the wave function triad. The original positions of $\boldsymbol{m}, \boldsymbol{n}$, and $\boldsymbol{s}$ are along the axes $x, y$, and $z$ respectively. The first rotation by the angle $\varphi$ is in the plane $x y$, which brings the first two vectors to the positions $\boldsymbol{m}_{1}$ and $\boldsymbol{n}_{1}$. The second rotation by the angle $\beta$ is in the plane confining the axis $z$ and the vector $\boldsymbol{m}_{1}$ (around the vector $\boldsymbol{n}_{1}$ ). This brings the vector $\boldsymbol{s}$ to its final position and rotates the vector $\boldsymbol{m}_{1}$ to $\boldsymbol{m}_{2}$. The last third rotation by the angle $\alpha$ is around the vector $\boldsymbol{s}$, which transforms the vectors $\boldsymbol{m}_{2}$ and $\boldsymbol{n}_{1}$ to the final vectors $\boldsymbol{m}$ and $\boldsymbol{n}$ determined by Eq. (30).

Using the Euler angles as variables the momentum flux tensor is

$$
\Pi_{i j}=\rho v_{i} v_{j}+\frac{\hbar^{2} \rho}{2 m^{2}}\left(\nabla_{i} \beta \nabla_{j} \beta+\sin ^{2} \beta \nabla_{i} \varphi \nabla_{j} \varphi\right)+P \delta_{i j},
$$

where the pressure $P$ is equal to the Lagrangian determined by Eq. (21).

Up to now our equations were isotropic in the spin space of the vector $\boldsymbol{s}$. But in an isotropic ferromagnet vortices as stable linear topological defects do not exist, as well as neither mass nor spin superfluidity is possible. Thus we shall add to our Hamiltonian terms breaking spherical symmetry but still invariant with respect to rotations around the axis $z$ (uniaxial anisotropy):

$$
H_{A}=-\gamma H_{e f} S s_{z}+\frac{\rho G s_{z}^{2}}{2} .
$$

Here $\gamma$ is the gyromagnetic ratio. The first term linear in $s_{z}$ is the Zeeman energy. The field $H_{e f}$ can be an external magnetic field but not necessarily. Processes violating the conservation law of spin usually are weak in comparison with the exchange interaction. 
Pumping magnons one can create a non-equilibrium $z$ component of spin, which relaxes quite slowly, and this relaxation can be compensated by continuing magnon pumping. With good accuracy one may consider this state as a quasi-equilibrium state with fixed $z$ component of spin. Such states under the name magnon BEC were realized both in solids [21] and in ferromagnetic spin-1 BEC [22]. Then $H_{e f}$ is a Lagrange multiplier, which determines the value of fixed total spin. The second term in Eq. (35) is called in magnetism easy-axis $(G<0)$ or easy-plane $(G>0)$ anisotropy. In the theory of cold atoms they call it the quadratic Zeeman energy [1]. The anisotropy energy determines two possible phases with the orientational phase transition between them. At $\gamma S H_{e f}>\rho G$ the energy is minimal at $s_{z}=1$ (easy-axis phase), while at $\gamma S H_{e f}<\rho G$ the spin is confined in the plane parallel to the $x y$ plane and corresponding to $s_{z}=\gamma S H_{e f} / \rho G$ (easy-plane phase). Since in the easyplane phase invariance with respect to rotations around the axis $z$ is spontaneously broken, it is called also broken-axisymmetry phase [1].

Further we consider the case of incompressible liquid, when it is enough to analyze only soft spin modes and to neglect density variation. Using the Euler angles for the unit vector $s$ as in Eq. (30), the spin Hamiltonian including the anisotropy terms is

$$
\begin{array}{r}
H=\rho\left\{\frac{v_{s}^{2}}{2}+\frac{\hbar^{2}}{4 m^{2}}\left[\sin ^{2} \beta(\nabla \varphi)^{2}+(\nabla \beta)^{2}\right]+\frac{G\left(\cos \beta-s_{0}\right)^{2}}{2}\right\} \\
=\frac{\rho \hbar^{2}}{m^{2}}\left[\frac{(\nabla \alpha+\cos \beta \nabla \varphi)^{2}}{2}+\frac{\sin ^{2} \beta(\nabla \varphi)^{2}+(\nabla \beta)^{2}}{4}+\frac{m^{2} G\left(\cos \beta-s_{0}\right)^{2}}{2 \hbar^{2}}\right],
\end{array}
$$

where

$$
s_{0}=\frac{\gamma S H_{e f}}{\rho G}=\frac{\gamma \hbar H_{e f}}{m G},
$$

and the superfluid velocity satisfies the incompressibility condition

$$
\boldsymbol{\nabla} \cdot \boldsymbol{v}_{s}=-\boldsymbol{\nabla} \cdot(\boldsymbol{\nabla} \alpha+\cos \beta \boldsymbol{\nabla} \varphi)=0
$$

The equations of spin dynamics in polar angles are

$$
\begin{gathered}
\dot{\beta}+\left(\boldsymbol{v}_{s} \cdot \boldsymbol{\nabla}\right) \beta=\frac{\hbar \sin \beta}{2 m} \nabla^{2} \varphi-\frac{\hbar \cos \beta}{m} \boldsymbol{\nabla} \varphi \cdot \boldsymbol{\nabla} \beta, \\
\dot{\varphi}+\left(\boldsymbol{v}_{s} \cdot \boldsymbol{\nabla}\right) \varphi=-\frac{\hbar}{2 m}\left[(\boldsymbol{\nabla} \varphi)^{2} \cos \beta-\frac{\nabla^{2} \beta}{\sin \beta}\right]+\frac{m G\left(\cos \beta-s_{0}\right)}{\hbar} .
\end{gathered}
$$




\section{DYNAMICS OF VORTICES IN THE LLG THEORY FOR LOCALIZED SPINS}

Although our final goal is dynamics of vortices in the ferromagnetic spin-1 BEC, it is useful for later discussion and comparison to start from magnetic vortices in ferromagnetic insulators, where spin carriers are localized and the degree of freedom of motion of the medium as a whole is absent. The original LLG theory referred exactly to this case. The Lagrangian and the Hamiltonian of the LLG theory in angle variables are

$$
\begin{gathered}
\mathcal{L}=\frac{\hbar}{m} \rho_{1} \cos \beta \frac{\partial \varphi}{\partial t}-H \\
H=\frac{\hbar^{2} \rho_{2}}{4 m^{2}}\left[\sin ^{2} \beta(\nabla \varphi)^{2}+(\nabla \beta)^{2}\right]+\frac{\rho_{2} G\left(\cos \beta-s_{0}\right)^{2}}{2} .
\end{gathered}
$$

The equations of spin dynamics are

$$
\begin{gathered}
\dot{\beta}=\frac{\hbar \rho_{2} \sin \beta}{2 m \rho_{1}} \nabla^{2} \varphi-\frac{\hbar \rho_{2} \cos \beta}{m \rho_{1}} \nabla \varphi \cdot \nabla \beta \\
\dot{\varphi}=-\frac{\hbar \rho_{2}}{2 m \rho_{1}}\left[(\nabla \varphi)^{2} \cos \beta-\frac{\rho_{2} \nabla^{2} \beta}{\sin \beta}\right]+\frac{m \rho_{2} G\left(\cos \beta-s_{0}\right)}{\hbar \rho_{1}} .
\end{gathered}
$$

Here we introduced the densities $\rho_{1}$ and $\rho_{2}$. The density $\rho_{1}$ in the first term of the Lagrangian (the Wess-Zumino term) determines the spin density $S=\hbar \rho_{1} / m$, which is a constant in the LLG theory. The second density $\rho_{2}$ determines phase stiffness of the magnetic order parameter. In the Heisenberg model of ferromagnetic insulators $\rho_{2}$ is proportional to the exchange interaction between spins at neighboring sites. Introduction of the densities $\rho_{1}$ and $\rho_{2}$ makes comparison of the LLG theory for ferromagnetic insulators and spin-1 BEC more convenient: if $\rho_{1}$ and $\rho_{2}$ are equal to the total mass density $\rho$ these equations coincide with Eqs. (39) and (40) but without $\boldsymbol{v}_{s}$-dependent terms.

Without anisotropy the order parameter space is $S_{2}$, which is a $2 \mathrm{D}$ surface of a unit sphere in the 3D space. Every point of $S_{2}$ corresponds to some direction of the vector $\boldsymbol{s}$. Spin superfluidity and vortices are possible only in the easy-plane phase when the order parameter space reduces to a circumference of the sphere $S_{2}$ corresponding to some fixed value of $s_{z}\left(\left|s_{z}\right|<1\right)$. But only periphery of the vortex very far from its axis maps on this circumference. The core of the vortex maps on an upper (northern) or lower (southern) part of the sphere. The vortex is characterized by two topological numbers [15]. The first one is the winding number, i.e., the number of rotations the spin makes on going around a vortex 


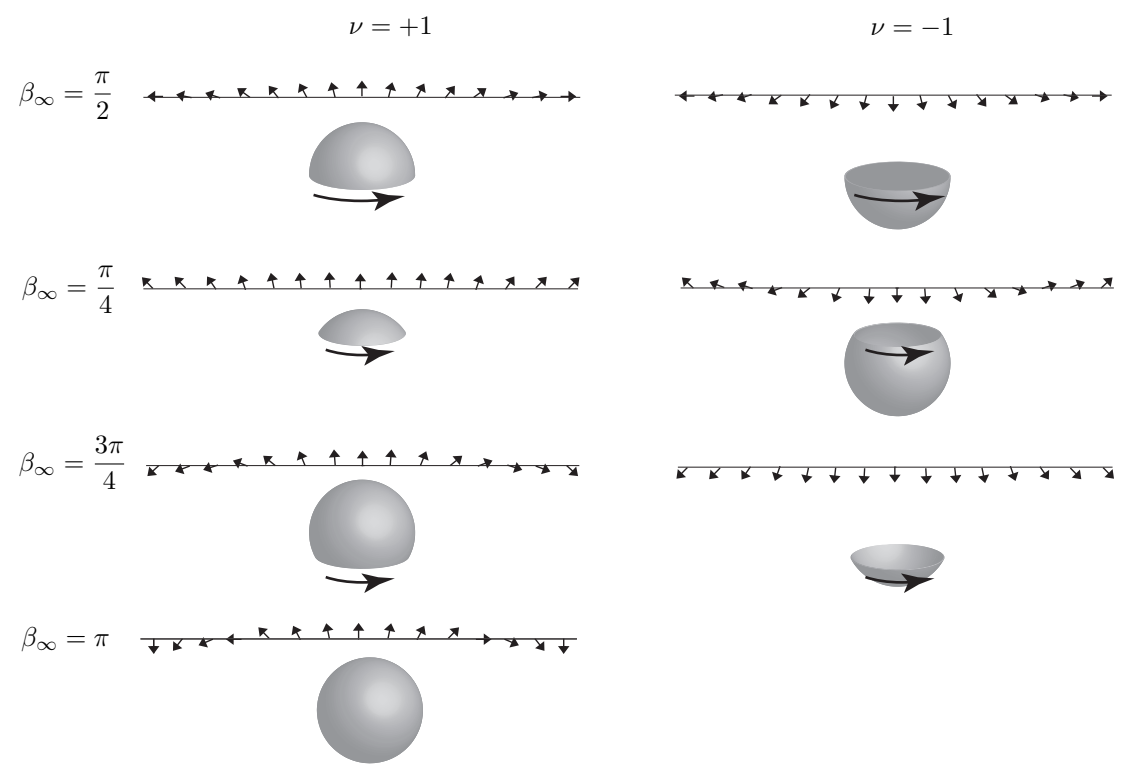

FIG. 2. Spin vectors $s$ in axial cross-sections of skyrmion cores and mapping on the space $S_{2}$ for vortex states with polarizations $\nu= \pm 1$ and polar angles $\beta_{\infty}=\pi / 4, \pi / 2,3 \pi / 4$, and $\pi$. Larger arrows show direction of circular spin currents around the vortex (skyrmion) axis.

(the analogue of the number of circulation quanta for a vortex in superfluid hydrodynamics). The second number, which can be called polarization, takes two values $\nu= \pm 1$. Two signs correspond to a sign of the spin component $s_{z}$ at the vortex axis. We choose direction of the axis $z$ so that the in-plane spin component rotates counterclockwise around it. The positive polarization corresponds to mapping on the northern part of the sphere, while the negative polarization points out mapping on the southern one. Mapping of vortex states with two polarizations and various values of the polar angle $\beta_{\infty}$ far from the vortex are shown in Fig. 2. The vortex core at $\beta_{\infty}=\pi$ has the structure of a skyrmion with the charge $Q=1$. The skyrmion charge is a measure of wrapping of the spin vector around the sphere $S_{2}$ and equal to $Q=\sin ^{2} \frac{\beta_{\infty}}{2}$. At $\beta_{\infty}=\pi / 2$ when at periphery the spin is confined in the $x y$ plane, the core skyrmion is a meron, or a half-skyrmion with the skyrmion charge one-half [23]. Other values of $\beta_{\infty}$ correspond to other fractional skyrmion charges. Thus in the presence of uniaxial anisotropy the skyrmion charge is not quantized and may vary continuously.

Skyrmions shown in Fig. 2 are Neel skyrmions with non-zero magnetostatic charges proportional to $\boldsymbol{\nabla} \cdot \boldsymbol{s}$. But rotation in the spin space around the axis $z$ transforms skyrmions to Bloch skyrmions. Our model is invariant with respect to this rotation since it ignores the 
magnetostatic interaction.

In a straight axisymmetric vortex spin depends only on two polar coordinates $r, \phi$. For a single-quantum vortex $\varphi$ does not depend on $r$ and is equal to the azimuthal angle $\phi$. The gradient of the spin phase $\varphi$,

$$
\nabla \varphi=\frac{[\hat{z} \times \boldsymbol{r}]}{r^{2}}
$$

has only the azimuthal component $\propto 1 / r$. The polar angle $\beta$ depends only on $r$. Then the Hamiltonian (42) does not depend on the angle $\phi$ and becomes

$$
H=\rho_{2}\left\{\frac{\hbar^{2}}{4 m^{2}}\left[\frac{\sin ^{2} \beta}{r^{2}}+\left(\frac{d \beta}{d r}\right)^{2}\right]+\frac{G\left(\cos \beta-s_{0}\right)^{2}}{2}\right\} .
$$

The Euler-Lagrange equation for this Hamiltonian describes spin texture in a resting vortex:

$$
\frac{d^{2} \beta}{d r^{2}}+\frac{1}{r} \frac{d \beta}{d r}-\sin \beta\left(\frac{\cos \beta}{r^{2}}-\frac{\cos \beta-s_{0}}{\xi^{2}}\right)=0
$$

where

$$
\xi=\frac{\hbar}{m \sqrt{2 G}}
$$

and $s_{0}=\cos \beta_{\infty}$ is the value of $s_{z}$ at large distances from the vortex axis. At small $r \beta \propto r$, while at large $r \beta$ approaches to the equilibrium value $\beta_{\infty}$ :

$$
\beta \approx \beta_{\infty}-\frac{\xi^{2} \cos \beta_{\infty}}{r^{2} \sin \beta_{\infty}}
$$

One can define the core radius as a distance $r$ at which the correction to the asymptotic value $\beta_{\infty}$ becomes comparable with $\beta_{\infty}$ itself. This yields the core radius of the order $r_{c} \sim \xi$ excepting very small $\beta_{\infty}$, when Eq. 47) becomes

$$
\frac{d^{2} \beta}{d r^{2}}+\frac{1}{r} \frac{d \beta}{d r}-\frac{\beta}{r^{2}}-\frac{\left(\beta^{2}-\beta_{\infty}^{2}\right) \beta}{2 \xi^{2}}=0
$$

This equation is identical to the Gross-Pitaevskii equation for radial distribution of the density of the vortex in a single-component superfluid. It determines the core radius as $r_{c} \sim \xi / \beta_{\infty}$, which diverges at $\beta_{\infty} \rightarrow 0$.

In the easy-axis phase $\left[s_{0}>0\right.$ in Eqs. (36) and (46)] there is no magnetic vortices with circular spin currents at large distances. However, skyrmion with the charge $Q=1\left(\beta_{\infty}=\pi\right)$ is still possible and shown in Fig. 2. Without anisotropy [ $\xi \rightarrow \infty$ in Eq. (47)] spatial rescaling does not change the energy of the $Q=1$ skyrmion and it can have any size. At fixed scaleinvariant distribution of $\beta$ the easy-axis anisotropy energy is smaller at smaller skyrmion 
size, and the skyrmion is expected to collapse to very small size. But anisotropy modifies this distribution, and this could stabilize the skyrmion at the scale $\xi$. Numerical calculation [6] showed that the vortex with the $Q=1$ skyrmion core is unstable in ferromagnetic insulators discussed in the present section, but is stable in the ferromagnetic spin-1 BEC (see below). In ferromagnetic insulators the $Q=1$ skyrmion can be stabilized by other interactions, e.g., by gradient terms of higher order [24, 25], or by the Dzyaloshinskii-Moriya interaction [26].

We consider the quasimomentum balance in the coordinate frame moving with constant vortex velocity $\boldsymbol{v}_{L}$, calculating the total quasimomentum flux through cylindrical surfaces restricting the area around the vortex. All time derivatives are $\partial / \partial t=-\left(\boldsymbol{v}_{L} \cdot \boldsymbol{\nabla}\right)$, and the quasimomentum flux tensor in the moving frame is

$$
\Pi_{i j}=\frac{\hbar^{2} \rho_{2}}{2 m^{2}}\left(\nabla_{i} \beta \nabla_{j} \beta+\sin ^{2} \beta \nabla_{i} \varphi \nabla_{j} \varphi\right)-g_{i} v_{L j}+P \delta_{i j},
$$

where the quasimomentum density is

$$
\boldsymbol{g}=-\frac{\partial \mathcal{L}}{\partial \dot{\varphi}} \boldsymbol{\nabla} \varphi=-\frac{\hbar}{m} \rho_{1} \cos \beta \boldsymbol{\nabla} \varphi
$$

The quasipressure $P$ is equal to the Lagrangian 41). We expand the expressions for $P$ and $\Pi_{i j}$ in small deviations $\beta^{\prime}$ and $\boldsymbol{\nabla} \varphi^{\prime}$ from values of the polar angle $\beta$ and the gradient $\boldsymbol{\nabla} \varphi$ in the stationary vortex. Only terms proportional to $\boldsymbol{v}_{L}$ and to the constant phase gradient deviation $\nabla \varphi^{\prime}$ at large distances from the vortex axis, which is connected with the spin current past the vortex,

$$
\boldsymbol{j}_{z}=-\frac{\hbar^{2} \rho_{2}}{2 m^{2}} \sin ^{2} \beta_{\infty} \nabla \varphi^{\prime}
$$

are important for the quasimomentum balance. Correction to the quasimomentum flux is $\Pi_{i j}^{\prime}=\frac{\hbar^{2} \rho_{2}}{2 m^{2}} \sin ^{2} \beta \nabla_{i} \varphi \nabla_{j} \varphi^{\prime}-v_{L j} g_{i}+P^{\prime} \delta_{i j}=\frac{\hbar^{2} \rho_{2}}{2 m^{2}} \sin ^{2} \beta \nabla_{i} \varphi \nabla_{j} \varphi^{\prime}+\frac{\hbar \rho_{1}}{m} \cos \beta \nabla_{i} \varphi v_{L j}+P^{\prime} \delta_{i j}$,

where the quazipressure perturbation is

$$
P^{\prime}=-\frac{\hbar \rho_{1}}{m} \cos \beta\left(\boldsymbol{v}_{L} \cdot \boldsymbol{\nabla}\right) \varphi-\frac{\hbar^{2} \rho_{2}}{2 m^{2}} \sin ^{2} \beta\left(\boldsymbol{\nabla} \varphi \cdot \boldsymbol{\nabla} \varphi^{\prime}\right) .
$$

In the LLG theory the quasimomentum density $\boldsymbol{g}$ given by Eq. 52 diverges at the axis of the vortex. Because of it the total variation of the quasimomentum in the area around the vortex is determined by the quasimomentum flux not only through the surface at large 
distances $r$ but also through the surface at very small distances $r$ from the vortex axis [15]:

$$
\int_{r \rightarrow \infty} \Pi_{i j} d S_{j}-\int_{r \rightarrow 0} \Pi_{i j} d S_{j}=-\frac{2 \pi \hbar}{m}\left\{\rho_{1}\left(1-\cos \beta_{\infty}\right)\left[\hat{z} \times \boldsymbol{v}_{L}\right]_{j}+\frac{\rho_{2} \sin ^{2} \beta_{\infty}}{2}\left[\hat{z} \times \nabla \phi^{\prime}\right]_{j}\right\}=0 .
$$

The quasimomentum balance equation is at the same time the force balance equation. The term proportional to the vortex velocity $\boldsymbol{v}_{L}$ is a gyroscopic force similar to the Magnus force on the hydrodynamic vortex. The second term in the right-hand side is a force produced by a spin supercurrent past the vortex (analog of the Lorentz force on the vortex in superconductors and superfluids). The Lorentz force is a gradient of the energy of interaction between the vortex and the spin current past the vortex. This energy is determined by cross terms containing the phase gradient (45) induced by the vortex and the phase gradient $\boldsymbol{\nabla} \varphi^{\prime}$

produced by the spin current. We assumed that the spin current is constant far from the vortex line. But in general it can vary at scales essentially exceeding the vortex core radius, taking into account phase variation induced by other distant vortices. In this case Eq. (56) contains $\boldsymbol{\nabla} \varphi^{\prime}$ at distances much larger than the vortex core radius, but much smaller than the distance from other vortices.

Equation (56) yields the relation between the vortex velocity $\boldsymbol{v}_{L}$ and the spin current $\boldsymbol{j}_{z}$. Up to now we considered the vortex with positive polarization and one $2 \pi$-rotation of the spin $s$ around the vortex axis. Generalizing for arbitrary polarization $\nu= \pm 1$ and arbitrary integer number $n$ of rotation of $s$ :

$$
\boldsymbol{v}_{L}=-\frac{n\left(\nu+\cos \beta_{\infty}\right)}{2} \frac{\rho_{2}}{\rho_{1}} \nabla \phi^{\prime}=\frac{n m^{2}}{\rho_{1} \hbar^{2}\left(\nu-\cos \beta_{\infty}\right)} \boldsymbol{j}_{z}
$$

\section{DYNAMICS OF VORTICES IN THE FERROMAGNETIC SPIN-1 BEC}

In an axisymmetric vortex with a single quantum of circulation of the spin phase $\varphi$ the azimuthal velocity around the vortex axis in general is

$$
v_{s}(r)=\frac{\hbar[N-\cos \beta(r)]}{m r} \text {. }
$$

Here the integer $N$ points out the number of full $2 \pi$ rotations of the Euler angle $\alpha$ around the vortex axis [see Eq. (31)]. This velocity satisfies the Mermin-Ho theorem connecting the velocity with variation of $\boldsymbol{s}$ [the term $\propto \cos \beta(r)]$. 
Both contributions are singular at $r \rightarrow 0$. However, we look only for nonsingular vortices, with the energy smaller than singular ones. Two singular contributions to the velocity cancel one another if $N=1$ for the vortex with positive polarization and $N=-1$ for the vortex with negative polarization.

Taking into account Eq. (58) with $N=1$ (positive polarization) the Hamiltonian (36) for axisymmetric vortex becomes

$$
H=\rho\left\{\frac{\hbar^{2}}{4 m^{2}}\left[\frac{(2-\cos \beta)^{2}-1}{r^{2}}+\left(\frac{d \beta}{d r}\right)^{2}\right]+\frac{G\left(\cos \beta-s_{0}\right)^{2}}{2}\right\} .
$$

The Euler-Lagrange equation for this Hamiltonian is

$$
\frac{d^{2} \beta}{d r^{2}}+\frac{1}{r} \frac{d \beta}{d r}-\sin \beta\left(\frac{2-\cos \beta}{r^{2}}-\frac{\cos \beta-s_{0}}{\xi^{2}}\right)=0
$$

where $s_{0}=\cos \beta_{\infty}<1$ in the easy-plane phase. In the theory of the A phase of superfluid ${ }^{3}$ He the vortex at $\beta_{\infty}=\pi / 2$ (meron) was known as Mermin-Ho vortex, while the vortex at $\beta_{\infty}=\pi$ was called the Anderson-Toulouse vortex [16]. This vortex has circulation of $\boldsymbol{v}_{s}$ but no circulating spin current far from the vortex. All other vortices at $\beta_{\infty}<\pi$ have both circulations. In contrast to ferromagnetic insulators with the Euler-Lagrange equation (47), according to numerical solution of Eq. (60) [6], anisotropy is able to stabilize the skyrmion with the charge $1\left(\beta_{\infty}=\pi\right)$ [see discussion below Eq. (50)].

The ferromagnetic spin-1 BEC is Galilean invariant, and the quasimomentum does not differ from true momentum. As in the previous section, we consider the momentum balance in the coordinate frame moving with the velocity $\boldsymbol{v}_{L}$ and expand the momentum flux tensor $\Pi_{i j}$ in small perturbations produced by vortex motion and currents past the vortex:

$$
\Pi_{i j}^{\prime}=\rho v_{s i}\left(v_{j}-v_{L j}\right)+\frac{\hbar^{2} \rho}{2 m^{2}} \sin ^{2} \beta \nabla_{i} \varphi \nabla_{j} \varphi^{\prime}+P^{\prime} \delta_{i j}
$$

where the pressure perturbation is determined from the Bernoulli law:

$$
P^{\prime}=\frac{\hbar \rho}{m}\left[\left(\boldsymbol{v}_{L}-\boldsymbol{v}_{s}\right) \cdot \nabla\right] \theta-\frac{\hbar^{2} \rho}{2 m^{2}} \sin ^{2} \beta \nabla \varphi \nabla \varphi^{\prime} .
$$

In contrast to the LLG theory for localized spins, there is no terms in the momentum flux tensor divergent at the vortex axis. Therefore the variation of the total momentum around the vortex is determined only by the momentum flux through the surface far away from the vortex axis:

$$
\int_{r \rightarrow \infty} \Pi_{i j} d S_{j}=\frac{2 \pi \hbar \rho}{m}\left\{\left(1-\cos \beta_{\infty}\right)\left[\hat{z} \times\left(\boldsymbol{v}_{s}-\boldsymbol{v}_{L}\right)\right]_{j}-\frac{\sin ^{2} \beta_{\infty}}{2}\left[\hat{z} \times \nabla \phi^{\prime}\right]_{j}\right\}=0 .
$$


Generalizing this equation on the vortex with polarization $\nu= \pm 1$ and integer number $n$ of $2 \pi$-rotations of $\boldsymbol{s}$ around the $z$ axis the vortex velocity is

$$
\boldsymbol{v}_{L}=\boldsymbol{v}_{s}-\frac{n\left(\nu+\cos \beta_{\infty}\right)}{2} \boldsymbol{\nabla} \phi^{\prime}=\frac{\boldsymbol{j}}{\rho}+\frac{n m}{\hbar \rho\left(\nu-\cos \beta_{\infty}\right)} \boldsymbol{j}_{z} .
$$

This is a generalization of Helmholtz's theorem, which tells that in a scalar superfluid (or an ideal fluid in classical hydrodynamics) the vortex moves with the fluid velocity $\boldsymbol{v}_{s}$. In the ferromagnetic spin-1 BEC not only the mass current $\boldsymbol{j}$ but also spin current $\boldsymbol{j}_{z}$ produces the Lorentz force driving the vortex. The transverse gyroscopic force (Magnus force in hydrodynamics) is

$$
\boldsymbol{F}_{G}=-\frac{2 \pi n \hbar}{m}\left(\nu-\cos \beta_{\infty}\right) \rho\left[\hat{z} \times \boldsymbol{v}_{L}\right]
$$

The force is proportional to the circulation of the superfluid velocity $\oint \boldsymbol{v}_{s} \cdot d \boldsymbol{l}=(n h / m)(\nu-$ $\left.\cos \beta_{\infty}\right)$. It is interesting that the gyrotropic force in ferromagnetic insulator [see Eq. (56)] is given by a similar expression (apart from the difference between two densities $\rho$ and $\rho_{1}$ ), although there is no superfluid velocity in the theory. On the other hand, the superfluid circulation is proportional to the skyrmion charge in the vortex core, which is present in the both theories. Thus, a more careful statement is that the gyroscopic force is proportional to the core skyrmion charge.

\section{DISCUSSION AND CONCLUSIONS}

We analyzed dynamics of nonsingular vortices in a ferromagnetic spin-1 BEC, where both mass and spin superfluidity are possible in the presence of uniaxial anisotropy. Vortices are nonsingular only if there is circulation of the wave function phase and the spin phase (the angle of spin rotation around a chosen axis). Their cores have structure of skyrmions with charges tuned by uniaxial anisotropy.

The equation of vortex motion is derived from the quasimomentum conservation law following from Noether's theorem for translationally invariant media. The ferromagnetic

spin-1 BEC is Galilean invariant, and the quasimomentum does not differ from the true momentum. Vortex dynamics in a ferromagnetic spin-1 BEC is compared with dynamics of magnetic vortices following from the LLG theory for ferromagnetic insulators. In the latter case the vortex is driven by the spin current past the vortex, while in the former one both the mass and the spin currents make the vortex to move. In the both cases the driving 
force (Lorentz force in superfluid hydrodynamics) is balanced by the transverse gyrotropic force proportional to the vortex velocity $\boldsymbol{v}_{L}$ (analog of the Magnus force in scalar superfluids and classical ideal fluids). The gyrotropic force is proportional to a charge of a skyrmion emerging in a vortex core.

In a ferromagnetic spin-1 BEC the core skyrmion charge determines circulation of the superfluid velocity. On the other hand, the frequencies of vortex precession in a potential trap or of Kelvin waves along vortex lines are proportional to the superfluid circulation [19]. This can be used for experimental check of the results of the present analysis.

[1] Y. Kawaguchi and M. Ueda, Phys. Rep. 520, 253 (2012).

[2] E. B. Sonin, Adv. Phys. 59, 181 (2010).

[3] H. Chen and A. H. MacDonald, in Universal themes of Bose-Einstein condensation, edited by N. Proukakis, D. Snoke, and P. Littlewood (Cambridge University Press, 2017) Chap. 27, pp. 525-548, arXiv:1604.02429.

[4] L. D. Landau and E. M. Lifshitz, Statistical physics. Part II (Pergamon Press, 1980).

[5] A. Lamacraft, Phys. Rev. A 77, 063622 (2008).

[6] E. B. Sonin, "Spin and mass superfluidity in ferromagnetic spin-1 BEC," (2018), arXiv:1801.01099.

[7] E. B. Sonin, Phys. Rev. B 95, 144432 (2017).

[8] L. Onsager, Nuovo Cimento Suppl. 6, 249 (1949).

[9] R. P. Feynman, in Progress in Low Temperature Physics, Vol. 1, edited by C. J. Gorter (NorthHolland, 1955) pp. 17-53.

[10] A. Kosevich, V. Voronov, and I. Manzhos, Zh. Eksp. Teor. Fiz. 84, 148 (1983), [Sov. Phys.JETP, 57, 86-92 (1983)].

[11] A. M. Kosevich, B. A. Ivanov, and A. S. Kovalev, Nonlinear magnetization waves, dynamic and topological solitons (Naukova Dumka Kiev, 1988) (in Russian).

[12] A. M. Kosevich, B. A. Ivanov, and A. S. Kovalev, Phys. Rep. 194, 117 (1990).

[13] A. Thiele, Phys. Rev. Lett. 30, 230 (1973).

[14] D. Huber, Phys. Rev. B 26, 3758 (1982).

[15] A. V. Nikiforov and E. B. Sonin, Zh. Eksp. Teor. Fiz. 85, 642 (1983), [Sov. Phys.-JETP, 58, 
373-378 (1983)].

[16] M. M. Salomaa and G. E. Volovik, Rev. Mod. Phys. 59, 533 (1985), erratum: 60, 573 (1988).

[17] E. B. Sonin, Pis'ma Zh. Eksp. Teor. Fiz. 43, 601 (1986), [JETP Lett., 43, 779-782 (1986)].

[18] N. D. Mermin and T. L. Ho, Phys. Rev. Lett. 36, 594 (1976).

[19] E. B. Sonin, Dynamics of quantised vortices in superfluids (Cambridge University Press, 2016).

[20] E. B. Sonin, Zh. Eksp. Teor. Fiz. 87, 1670 (1984), [Sov. Phys.-JETP, 60, 959-967 (1984)].

[21] S. O. Demokritov, V. E. Demidov, O. Dzyapko, G. A. Melkov, A. A. Serga, B. Hillebrands, and A. N. Slavin, Nature 443, 430 (2006).

[22] F. Fang, R. Olf, S. Wu, H. Kadau, and D. M. Stamper-Kurn, Phys. Rev. Lett. 116, 095301 (2016).

[23] H.-B. Braun, Advances in Physics 61, 1 (2012).

[24] B. Ivanov, V. Stephanovich, and A. Zhmudskii, J. Magn. Magn. Mater. 88, 116 (1990).

[25] A. Abanov and V. L. Pokrovsky, Phys. Rev. B 58, R8889 (1998).

[26] N. Nagaosa and Y. Tokura, Nat. Nanotechnol. 8, 899 (2013). 